\title{
ERRORES EN EL APRENDIZAJE DE LAS MATEMÁTICAS FINANCIERAS
}

\section{ERRORS IN LEARNING OF FINANCIAL MATHEMATICS}

\author{
Ana F. Carazo \\ (afercar@upo.es) \\ Raúl Brey \\ (rbresan@upo.es) \\ Departamento de Economía, Métodos Cuantitativos e Historia Económica \\ Universidad Pablo de Olavide, Sevilla
}

RESUMEN: En este artículo nos centramos en el estudio de los errores matemáticos de alumnos universitarios que cursan estudios en titulaciones de Economía y Administración y Dirección de Empresas. En el trabajo seguimos una propuesta empírico-inductiva para el análisis de los errores y su posterior identificación y clasificación, interpretándolos posteriormente en relación al conocimiento matemático-financiero que se pretende desarrollar. Los resultados muestran cómo algunos de los errores identificados están también presentes en resultados de estudios realizados con alumnos de secundaria, confirmando su persistencia y señalando su efecto sobre diferentes aspectos matemáticofinancieros. Además, hemos identificado otros errores, vinculados a contenidos como los números racionales y la dimensión temporal, que pueden afectar a la comprensión de ideas básicas en la asignatura de Matemáticas Financieras.

PALABRAS CLAVE: Matemáticas Financieras, Errores en el aprendizaje, Alumnos universitarios

\begin{abstract}
This article focuses on the study of mathematical errors made by university students who study Financial Mathematics as part of a degree in Economics or Business Administration. In this work, we adopt an empirical-inductive procedure for the analysis of the errors and their subsequent identification and classification, interpreting them later with respect to the knowledge of financial mathematics that it is intended to develop. The results have showed that some of the identified errors are also found in studies carried out with secondary students, confirming their persistence and pointing out their effects on different aspects of the subject of Financial Mathematics. In addition, we have identified other errors linked to contents such as the rational numbers and the temporal dimension, which may affect the understanding of basic ideas in the subject of Financial Mathematics.
\end{abstract}

KEY WORDS: Financial Mathematics, Errors in learning, University Students 


\section{INTRODUCCIÓN}

En los últimos años, la incorporación de las universidades españolas al Espacio Europeo de Educación Superior ha supuesto un profundo cambio en la educación universitaria, que afecta tanto a las titulaciones como a los contenidos o la metodología docente. Así, en el REAL DECRETO 1393/2007, de 29 de octubre, por el que se establece la ordenación de las enseñanzas universitarias oficiales se indica textualmente que

...la nueva organización de las enseñanzas universitarias responde no sólo a un cambio estructural sino que además impulsa un cambio en las metodologías docentes, que centra el objetivo en el proceso de aprendizaje del estudiante, en un contexto que se extiende ahora a lo largo de la vida.

(BOE número 260 de 30/10/2007, p. 44037)

destacándose por tanto explícitamente la importancia de los procesos de aprendizaje en los estudiantes universitarios.

Ahora bien, el asumir la importancia de dichos procesos de aprendizaje en los estudios superiores lleva a la necesidad de profundizar en todo aquello que puede afectarlos. En este sentido, los errores de los alumnos universitarios, considerados como 'indicadores' de lo que sucede en dichos procesos se convierten en algo a tener en cuenta por los profesores de estos niveles. En nuestro estudio, siguiendo a Socas (1997) asumimos que:

El error va a tener procedencias diferentes, pero, en todo caso, va a ser considerado como la presencia en el alumno de un esquema cognitivo inadecuado y no solamente como consecuencia de una falta específica de conocimiento o de un despiste (p. 125).

En este contexto, en este trabajo nos planteamos los siguientes objetivos:

- Identificar errores matemáticos frecuentes en estudiantes universitarios de licenciaturas y grados relacionados con economía y administración y dirección de empresas (ciencias empresariales, actuariales, finanzas y contabilidad, economía y marketing)

- Analizar las dificultades que dichos errores pueden originar en el desarrollo de conceptos y procedimientos relevantes en dichos estudios

Queremos señalar que este estudio no es en absoluto un 'estudio de déficit', en el que se trate de poner de manifiesto carencias de los estudiantes y se busquen responsabilidades en otros niveles educativos. Lo que aquí intentamos es aproximarnos a los diferentes errores que apreciamos en los trabajos de nuestros alumnos universitarios desde una doble perspectiva temporal: pasada, buscando su origen para poder ayudarles a solucionarlos, y futura, identificándolos como posibles causas de problemas en su futuro trabajo profesional.

\section{ENMARCANDO EL ESTUDIO}

Son muchos los trabajos que se han ocupado del estudio de los errores de los alumnos desde diferentes perspectivas, niveles educativos y contenidos matemáticos (entre muchos otros, y centrándonos en la presente década, podemos señalar los trabajos de Blanco, 2004; González-Martín y Camacho, 2005; Guillén, 2000). Centrándonos en aquellos estudios que aportan marcos que permiten abordar la clasificación y estudio de los errores matemáticos, en Rico (1995) se detallan diferentes propuestas planteadas por distintos autores. En particular, queremos mencionar el trabajo de Mosvshovitz-Hadar, Zaslavsky y Inbar (1987) que, basándose en una investigación sobre errores cometidos por alumnos de secundaria en matemáticas y desde una perspectiva que considera la naturaleza operacional de los 
errores, presentan seis categorías descriptivas de los errores que pueden ser utilizadas como modelo para su clasificación y que mencionamos a continuación:

1. Datos mal utilizados. Se incluyen en esta categoría los errores que pueden estar relacionados con diferencias entre los datos y el tratamiento que le da el alumno.

2. Interpretación incorrecta del lenguaje. Son errores que tienen su origen en una traducción incorrecta de hechos matemáticos descritos de un lenguaje a otro.

3. Inferencias inválidas lógicamente. Se sitúan aquí los errores que tienen que ver con razonamientos incorrectos y no con contenidos específicos.

4. Teoremas o definiciones distorsionados. Son errores que se producen por deformación de un principio, regla, teorema o definición.

5. Soluciones no verificadas. Su característica principal es que aunque cada paso en la realización de una tarea es correcto en sí mismo, el resultado final no es la solución a la pregunta planteada.

6. Errores técnicos. Se incluyen en esta categoría los errores de cálculo, manejo incorrecto de símbolos algebraicos y otros errores derivados de la ejecución de algoritmos.

Otros autores como Socas (1997), adoptando una perspectiva centrada en el origen de los errores, incorporan en su estudio componentes que van más allá de lo estrictamente matemático. En particular, este autor distingue en su trabajo tres ejes que pueden ayudar a situar los errores atendiendo a su origen: los que tienen su origen en un obstáculo, en ausencia de sentido y en actitudes afectivas y emocionales. Dentro de aquellos que tienen su origen en una ausencia de sentido, incluye tanto los que se relacionan con la complejidad de los objetos matemáticos como a los procesos de pensamiento matemático.

En el estudio que aquí presentamos nos centramos en la identificación de los errores matemáticos de los alumnos y su repercusión (si es que existe) en un ámbito profesional específico como es el campo de la economía y administración y dirección de empresas. En particular, de las cuatro líneas de investigación identificadas por Rico (1995) en relación a los errores (relativo al análisis y clasificación de los errores, causas de los mismos y clasificaciones; tratamiento curricular; estudios relacionados con la formación de profesores en relación a los errores que cometen los alumnos; trabajos de carácter técnico relacionados con una determinada clase de análisis sobre errores), nos situamos dentro de la primera línea. En nuestro trabajo se sigue una propuesta empírico-inductiva para su identificación y clasificación, interpretándolos posteriormente en relación al conocimiento matemático-financiero que pretendemos que nuestros alumnos desarrollen.

\section{METODOLOGÍA}

\section{El contexto}

Dentro de la titulación de diplomatura de Ciencias Empresariales y la licenciatura en Administración y Dirección de Empresas en nuestra universidad, en estos momentos las Matemáticas financieras son una asignatura anual de 9 créditos ( 90 horas). Se trata de una asignatura troncal que se cursa en segundo año. El objetivo general de la asignatura es proveer al alumno de los conocimientos y de la capacidad de razonamiento necesarios para desenvolverse en el mundo financiero y bancario. El objetivo particular es el estudio de las principales operaciones financieras, tales como capitalización, descuento bancario, operaciones de venta a plazos, amortización de préstamos o de empréstitos, entre otras. Por tanto, la materia que contiene la asignatura es importante para todo futuro profesional en economía, en administración y dirección de empresas, muy recomendable para alumnos especializados en Finanzas, y también para todos aquellos que piensen opositar a la Administración Pública o trabajar en Entidades Financieras y/o empresas aseguradoras. 
La formación matemática previa de los alumnos que cursan esta materia es diversa, ya que pueden proceder de distintos tipos de Bachilleratos, lo que implica haber cursado diferentes tipos de asignaturas de Matemáticas correspondientes a distintas posibilidades.

En la metodología de trabajo de la asignatura se incluyen unas hojas de problemas que deben ser completadas y entregadas por los alumnos en diferentes ocasiones a través del curso. Cada una de ellas contiene unos $2 / 3$ problemas sencillos que plantean en su enunciado situaciones lo más próximas posibles a las que se puedan encontrar nuestros alumnos en su futura labor profesional. Una vez resueltos, estos problemas son entregados al profesor para ser corregidos. En la figura 1 presentamos un ejemplo de los problemas que se incluyen en una de esas hojas. Junto a ello, los alumnos realizan los correspondientes exámenes reglados, en los que se les pide que resuelvan problemas similares aunque quizás un poco más completos que los que se les han ido proponiendo en las mencionadas hojas.

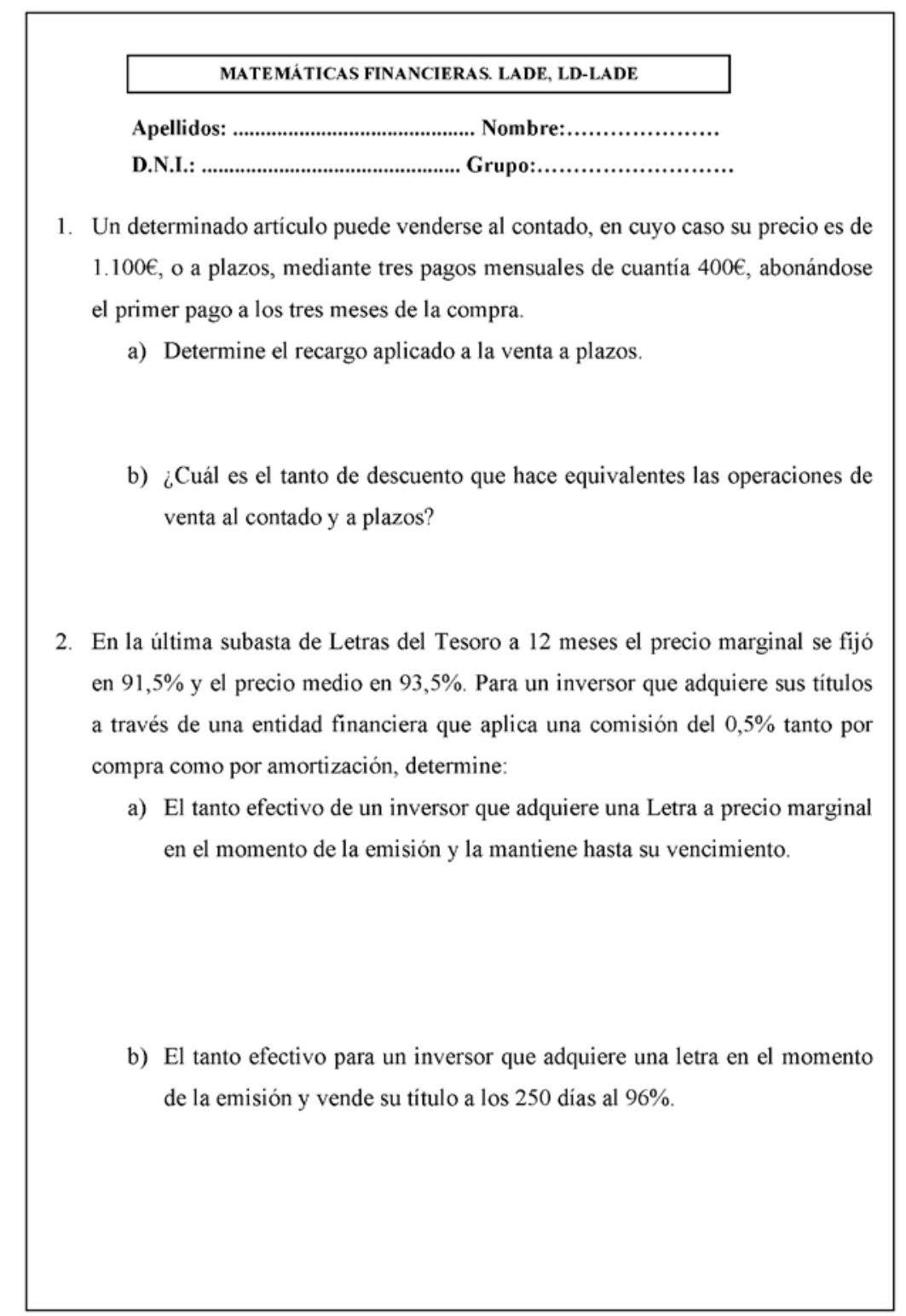

Fig 1. Ejemplo de «Hoja de problemas» 


\section{La recogida de datos}

Revisamos diferentes hojas de problemas y exámenes correspondientes a varios cursos escolares (2006/2007-2007/2008), seleccionamos aquellos trabajos en los que existía algún tipo de error (uno o más de uno). Estos fueron revisados cuidadosamente, comprobando si el error era sistemático, en el sentido de que se repetía el mismo tipo de error en diferentes problemas del mismo trabajo (hoja de problemas o examen), y descartándose aquellos errores que, por aparecer aislados, podían considerarse como accidentales para el alumno. Los errores repetidos correspondientes a un mismo alumno fueron considerados como un único error. Así, por ejemplo, en la respuesta de un alumno al segundo problema propuesto de los correspondientes a la figura 1, se consideró un mismo error susceptible de una posterior clasificación el que aparecía en las respuestas recogidas en la figura 2.

De este modo, se identificaron 300 errores que constituyen la fuente de datos de nuestro estudio.

2. En la última subasta de Letras del Tesoro a 12 meses el precio marginal se fijó en $91,5 \%$ y el precio medio en $93,5 \%$. Para un inversor que adquiere sus títulos a través de una entidad financiera que aplica una comisión del $0,5 \%$ tanto por compra como por amortización, determine:

a) El tanto efectivo de un inversor que adquiere una Letra a precio marginal en el momento de la emisión y la mantiene hasta su vencimiento.

$N=1000 €$
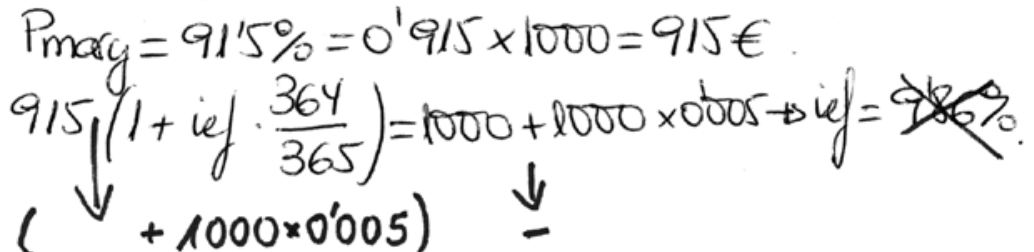

b) El tanto efectivo para un inversor que adquiere una letra en el momento de la emisión y vende su título a los 250 días al $96 \%$. Prta $=0^{\prime} 96 \times 1000=960 €$.

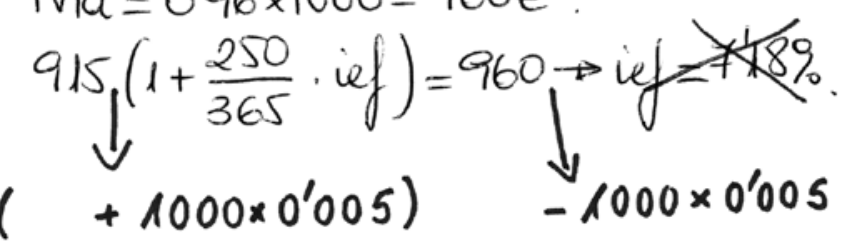

Fig. 2. Ejemplo de error correspondiente a las respuestas a la «Hoja de problemas» incluida en la figura 1

\section{Análisis de datos}

A partir de ahí, clasificamos cada uno de los errores siguiendo un proceso inductivo que constó de las siguientes fases:

- En primer lugar, analizamos los errores sistemáticos encontrados en cada uno de los trabajos de una manera cualitativa, tratando de buscar el origen de la respuesta de los alumnos e intentando hallar lógica que justificase sus respuestas 
- En segundo lugar, agrupamos los errores en base a su similitud, apoyándonos en el modelo proporcionado por Mosvshovitz-Hadar et al. (1987). Con este proceso no buscábamos ni incluir cada error en una categoría concreta de este autor ni una nueva categorización de los errores (que hubiese resultado muy parcial, dado lo limitado de la muestra y lo local de la situación planteada), sino un reconocimiento de unos orígenes comunes a los mismos.

- Finalmente, teniendo en cuenta la perspectiva adoptada en nuestro estudio, revisamos cada uno de los grupos considerados, atendiendo fundamentalmente a los diferentes contenidos matemático-financieros a los que afectaban.

En el apartado siguiente pasamos a describir los resultados obtenidos.

\section{RESULTADOS}

A continuación presentamos los tipos de errores identificados, especificando en cada apartado:

- qué incluimos en cada uno de ellos (descripción y características),

- el tanto por ciento o número de errores que se han situado en esa tipología,

- un ejemplo de los errores ahí situados, identificando con dos letras mayúsculas la codificación seguida (no el nombre del alumno). Queremos destacar que hemos optado por transcribir esos errores, no utilizándose una copia escaneada de los originales para respetar al máximo la privacidad.

\section{Errores identificados}

Dos grandes bloques de errores fueron identificados en el estudio. En un primer momento, teniendo en cuenta, como ya hemos mencionado, a Mosvshovitz-Hadar et al. (1987), fuimos identificando un primer bloque de errores que podríamos llamar 'transversales'; estos errores eran de distintas tipologías y afectaban a muy diferentes conceptos y procedimientos matemático-financieros. Sin embargo, según íbamos identificando estos errores, fuimos apreciando la existencia de un segundo grupo, que estaba relacionado con algún aspecto matemático concreto y con una incidencia mucho más focalizada sobre algo específico de la matemática financiera. Estos dos bloques van a ser desarrollados a continuación. Lógicamente, esta clasificación no pretende en absoluto ser dicotómica. Algunos errores situados en el segundo bloque podrían fácilmente haber sido situados en alguno de los apartados del primero, pero el objetivo de este trabajo no era tanto establecer una clasificación de errores como profundizar sobre los efectos de los mismos.

\section{a) Errores transversales que tienen incidencia en diferentes conceptos y procedimientos}

Se sitúan aquí aquellos errores que, por su carácter genérico, afectan a muy distintos ámbitos de los contenidos de las matemáticas financieras. En total de los 300 errores identificados, a este primer bloque corresponden 218 (72,67\%).

En la tabla siguiente incluimos los diferentes apartados identificados en este primer bloque $a$ ) y el número de errores encontrados correspondientes a cada uno de los subgrupos, inspirándonos para ello en la clasificación establecida por Mosvshovitz-Hadar et al. (1987). 


\begin{tabular}{|c|c|c|}
\hline \multicolumn{3}{|c|}{ a) ERRORES TRANSVERSALES } \\
\hline \multirow{2}{*}{$\begin{array}{l}\text { 1. Interpretación incorrecta } \\
\text { del lenguaje escrito }\end{array}$} & 1.1 Asociados globalmente al enunciado & 36 errores \\
\hline & 1.2 Asociados a palabras concretas & 60 errores \\
\hline $\begin{array}{l}\text { 2. Inferencias no válidas lógi- } \\
\text { camente }\end{array}$ & 2.1 Aplicar leyes de simple a compuesta y viceversa & 28 errores \\
\hline \multirow[b]{2}{*}{ 3. Memorización incorrecta } & 3.1 De formulaciones o expresiones matemáticas. & 8 errores \\
\hline & 3.2 De teoremas o definiciones mal recordados & 5 errores \\
\hline \multirow{3}{*}{$\begin{array}{l}\text { 4. Falta de verificación de la } \\
\text { solución }\end{array}$} & 4.1 No se explica el resultado o se explica de manera incorrecta & 6 errores \\
\hline & 4.2 El resultado final sin sentido aunque el procedimiento sea correcto & 6 errores \\
\hline & 4.3 Se da un resultado que no es lo que se pide & 6 errores \\
\hline \multirow{4}{*}{ 5. Errores técnicos } & 5.1 Error al despejar & 22 errores \\
\hline & $\begin{array}{l}\text { 5.2 Se aplica el procedimiento correcto, pero no se llega a despejar lo } \\
\text { pedido de la expresión resultante o bien se parte de una fórmula } \\
\text { matemática complicada y no se continúa }\end{array}$ & 19 errores \\
\hline & 5.3 Problemas con el tratamiento de los paréntesis & 16 errores \\
\hline & 5.4 Fallo de cálculo en el resultado final & 6 errores \\
\hline
\end{tabular}

A continuación, describimos cada uno de los grupos, incorporando un ejemplo de cada uno de los errores correspondientes.

\section{Interpretación o lectura incorrecta del lenguaje escrito}

Se incluyen aquí los errores relacionados tanto con la comprensión global del enunciado como con la comprensión de palabras concretas. Las dificultades de comprensión del lenguaje matemáticofinanciero ya han sido identificadas por autores como Ortega y Ortega (2000) y Fernández y Escribano (2005), que señalan además los problemas que presenta traducir este lenguaje a una modelización matemática. Esto podría deberse a que es un lenguaje técnico y en muchas ocasiones nuevo para ellos.

\subsection{Asociados globalmente al enunciado}

Son aquellos errores que pueden ser debidos a una mala interpretación del enunciado. Pensamos que diferentes errores detectados pueden tener este origen como, por ejemplo: Se concede un interés del $5 \%$ y se trabaja con un 5,5\% / Se dice que los 2 primeros años se paga 'a' y los diez restantes ' 2 veces a' y se pone en todos a / Se pide valor actual y se presenta el resultado del valor final, etc.

Queremos destacar que somos conscientes de que algunos de estos errores pueden ser debidos a un simple desconocimiento de lo que se está tratando, sustituyéndolo de manera premeditada por algo que se conoce; no obstante, pensamos que es importante tenerlos en cuenta.

1.2 Asociados a palabras concretas como:

Nominal (tanto en relación al concepto de valor nominal de un activo financiero, como a la tasa de interés nominal).

En relación a esta palabra apreciamos dos fallos típicos. Por un lado, confundir qué se entiende por valor nominal de un activo financiero y qué se entiende por otros valores de dicho activo, como puede ser el valor de mercado y, por otro lado, el error referente a la tasa de interés nominal. En este caso los alumnos suelen confundir la tasa de interés nominal con otras tasas de interés (efectiva anual, interés trimestral, etc.).

Un ejemplo de los errores incluidos en este apartado podría ser:

Se da como dato en el problema una tasa de interés nominal pagadera mensualmente $j_{12}=0,03$ y se pone en la respuesta que es una tasa de interés mensual: $i_{12}=0,03$ (MA).

Queremos indicar que realmente si: $j_{12}=0,03 \rightarrow i_{12}=\frac{0,03}{12}=0,0025=0,25 \% \neq 3 \%$ 
Comisión y el traslado de su significado a las situaciones económico-financieras.

Ejemplo de los errores incluidos en este apartado:

Se dice que el banco aplica una comisión del 1\% sobre el ingreso anual constante "a", esto es, el banco cobra por la gestión de la cuenta una comisión del $1 \%$ sobre las anualidades recibidas (asi cada año recibimos la anualidad menos la comisión sobre la anualidad), pero en la respuesta incorrecta se suma la comisión (DM).

Al no restar la comisión estaríamos recibiendo más dinero y no menos. Lo correcto es:

$$
\text { Ingreso anual }=a-a \cdot 0,01=0,99 a
$$

Este error es muy común en problemas relacionados con el cálculo del valor del precio de adquisición y amortización de ciertos activos financieros cuya gestión se realiza a través de un gestor o entidad financiera que cobra comisiones por cada movimiento del activo. Este será el caso, por ejemplo, de la adquisición de Letras del Tesoro, la cual tradicionalmente se realiza a través de un intermediario financiero que se encarga de la gestión y, como contrapartida, cobra una comisión por compra y/o adquisición y otra por venta y/o amortización. Supongamos que en este caso todas las comisiones son del $0,5 \%$ del valor nominal, el nominal de la Letra es de $1000 €$ y el precio medio de adquisición es por ejemplo de $935 €$, y nos pidiesen que calculásemos el precio de adquisición y el de amortización transcurrido un año. El alumno suele considerar que el precio de adquisición es de 935 unidades monetarias, mientras que realmente costaría un poco más (los 935 u.m. más lo que le pagamos al gestor o a la entidad financiera que se encarga del proceso, esto es: $935+0,005 \times 1000=940 €$ ) y el precio que se recibe al amortizar la letra no son los $1000 €$, ya que hay que pagarle algo al gestor que se encarga de todo el proceso; en este caso la comisión es de $5 €(0,005 \times 1000)$ y, por tanto, se recibe lo que conceden menos la comisión (esto es, 1000-5=995€). Los alumnos no terminan de tener claro cuando la comisión supone un mayor gasto o cuando un menor ingreso.

\section{Tasa de interés}

Existen también errores relacionados con la interpretación de a qué tasa nos referimos en una situación financiera cualquiera, ya que puede tratarse de una tasa de descuento, de interés pospagable, prepagable, simple, compuesta, o incluso si se trata de tasas trimestrales, mensuales, efectivos, nominales, etc.

Ejemplo de los errores incluidos en este apartado:

Se dice que se resuelva el cuadro de amortización de un préstamo concedido por 10 años, de anualidad constante concedido a una tasa de interés anual prepagable del $6 \%$ ( $z=6 \%)$. En este caso, (MS) lo resuelve como si se tratase de un préstamo de las mismas características pero de interés pospagable.

No es lo mismo que se conceda un préstamo a interés prepagable que a interés pospagable, ya que el primer interés, como su propio nombre indica, se cobra a principio de año (prepagable) y a final de año si fuese pospagable. Además, la equivalencia entre el interés prepagable ( $\mathrm{z}$ y, por tanto, el pospagable (i) es:

$$
i=\frac{z}{1-z} \rightarrow i=\frac{0,06}{1-0,06}=0,0638297 \approx 6,38 \% \text { y por tanto } i \neq z \rightarrow 6 \% \neq 6,38 \%
$$

Muchas veces los alumnos saben realizar las equivalencias entre las diferentes tasas pero no asocian a cuál se está haciendo referencia en un momento determinado. Quizás porque el vocabulario tradicio- 
nal no hace diferencias entre las tasas, o habitualmente sólo se hace referencia al tipo de interés anual pospagable y, por esto, ellos asocian todas las tasas a ésta, o intentan convertir cualquier tasa de interés a la misma.

Lenguaje relativo a los Tipos de pagos y Rentas (prepagables, pospagables, diferidas, perpetuas, etc.).

También existen errores relacionados con el conjunto de pagos o rentas, ya que no es lo mismo que decidamos devolver un préstamo mediante 10 pagos cada uno de ellos al final de los próximos 10 años (renta pospagable), que hacerlo mediante 10 pagos que se realizarán a principio de cada año (renta prepagable), o incluso comenzar a pagar esa deuda hoy o dentro de un tiempo (renta diferida).

Es común que se entienda que si alquilamos una vivienda empecemos a pagar inmediatamente a principio de mes, pero no lo tienen tan claro cuando se les dice que empiezan a pagar un alquiler de manera prepagable. Los alumnos suelen tener dificultades al trasladar el lenguaje cotidiano a lenguaje matemático, o al expresar éste mediante su correspondiente formulación matemática, teniendo problemas incluso en la representación de gráfica de esta información.

Ejemplo:

Se compra una televisión de alta definición y se firma un sistema de financiación consistente en: comenzar a pagarlo inmediatamente mediante 10 entregas mensuales prepagables de $100 €$ cada una de ellas, siendo el interés aplicable del 0,5\% mensual compuesto. Se pregunta cuál sería el precio de la televisión si lo pagase completamente en el momento inicial (MF).

Se resuelve el problema erróneamente a través de una renta constante y pospagable de la siguiente manera:

$$
100 \cdot \frac{1-(1+0,005)^{-10}}{0,005}=973,04 €
$$

Este error se suele producir porque los alumnos no entienden que prepagable significa pagos a principio de periodo, y no es lo mismo comenzar a pagar a principio de mes que guardar el dinero y pagarlo al final de cada uno de los 10 próximos meses.

La respuesta correcta es:

$$
100 \cdot \frac{1-(1+0,005)^{-10}}{0,005} \cdot(1+0,005)=977,90 €
$$

Gráficamente la diferencia sería:

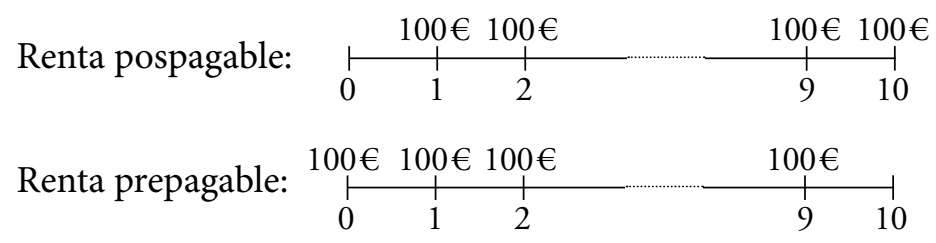

En general, podemos decir que la interpretación incorrecta del lenguaje figura dentro de las categorías descriptivas de errores propuestas por Movshovitz-Hadar et al. (1987). Así mismo, la importancia del lenguaje ha sido destacada por Socas (1997) como uno de los errores debidos a la complejidad de los objetos matemáticos. Nos parece del mayor interés señalar que, como indica este autor, en el caso del aprendizaje matemático-financiero estos errores se manifiestan también tanto en palabras específicas de este lenguaje (empréstito, pagaré, etc.), como en palabras mas cotidianas (tales como tasa, 
comisión, nominal, etc.) que pueden tener significados diferentes en el lenguaje habitual, lo que puede originar confusión.

\section{Inferencias no válidas lógicamente}

En este apartado hemos situado los errores debidos a un conocimiento anterior que proporciona unos modelos que no son válidos para la nueva situación planteada.

2.1 Aplicar leyes de simple a compuesta y viceversa.

Un caso muy frecuente en las matemáticas financieras es la utilización de manera incorrecta de leyes simples de capitalización y descuento cuando el enunciado del problema requiere de la utilización de leyes compuestas, y de compuestas cuando es necesario aplicar leyes simples. Ello implica un desconocimiento de la utilización de las leyes simple y compuesta y del momento en que se debe utilizar cada una de ellas.

Ejemplo:

El problema plantea una tasa de interés mensual compuesto $\left(i_{12}\right)$ pero el alumno trabaja con la formulación simple tanto en la transformación del interés $\left(i=\frac{i_{12}}{12}\right.$ ) como en el resto de resolución del ejercicio por lo que el resultado no es correcto (MR).

Según Socas (1997) se podrían considerar estos errores como asociados a procesos matemáticos previos que pueden producir dificultades en la construcción del conocimiento posterior.

\section{Memorización incorrecta}

La memorización incorrecta de procedimientos o fórmulas puede tener diferentes manifestaciones, tal y como se aprecia en los apartados siguientes (3.1 y 3.2):

3.1 Memorización incorrecta de formulaciones o expresiones matemáticas Ejemplo:

El alumno pone de memoria la fórmula del valor actual de una renta anual, constante y pospagable e inmediata de cuantía " $a$ " de " $"$ " años de duración en la que se aplica una tasa de interés anual pospagable (i). El alumno escribe la expresión matemática de dicho valor actual y se confunde en un signo (SR).

$$
\begin{aligned}
& \text { Por ejemplo, se pone } V A=a \cdot \frac{1-(1+i)^{n}}{i} \text { mientras lo correcto es poner }(-n), \\
& \text { esto es, } V A=a \cdot \frac{1-(1+i)^{-n}}{i} .
\end{aligned}
$$

Cabe destacar que en este tipo de expresiones es sencillo obtener la formulación de manera deductiva sin necesidad de tener que utilizar la memoria.

3.2 Teoremas o definiciones mal recordados.

Es muy común que se aprendan una fórmula de memoria y luego no saben qué significa cada uno de los términos de esta. Por lo tanto, no pueden sustituir los términos correctamente y no saben resolver el problema. En cualquier caso, en muchas ocasiones sustituyen por lo que sea para no dejarlo en blanco. 
Por ejemplo:

El alumno escribe correctamente la fórmula del valor actual de una progresión aritmética pero desconoce qué significa " $d$ " (que es la razón de crecimiento o decrecimiento de la progresión, por lo que no pueden sustituir su valor).

$$
V A=a \cdot \frac{1-(1+i)^{-n}}{i}+\frac{d}{i} \cdot\left(\frac{1-(1+i)^{-n}}{i}-n \cdot(1+i)^{-n}\right) \rightarrow i d ?
$$

Los errores incluidos en este apartado pueden ser considerados como pertenecientes a la categoría de teoremas o definiciones distorsionados identificada en alumnos de secundaria por MosvshovitzHadar et al. (1987). Para finalizar, somos conscientes de que en muchas ocasiones la memorización de conceptos o procedimientos puede ser necesaria, pero creemos que el problema está en aquellos casos en los que no se dota de significado a lo memorizado.

\section{Falta de verificación de la solución}

Incluimos aquí errores en los que la tarea planteada se realiza en forma correcta pero no se da respuesta a la pregunta planteada desde un punto de vista económico.

4.1 No explican el resultado o lo explican de manera incorrecta.

En estos casos, los alumnos tienen dificultades en interpretar desde el punto de vista económico-financiero el resultado como se puede apreciar en los siguientes ejemplos:

Ejemplo:

El alumno llega al final de la resolución de un ejercicio y termina diciendo, en este caso, que $n=16,65$ pero no explica qué significa esa solución.

En este caso sería que el pago final que completa la devolución de la deuda se realizará dentro de 16 años 7 meses y 24 días.

Ejemplo:

Al finalizar el ejercicio el alumno pone $n=5$ y dice que son 5 años.

En este último caso, el ejercicio se refería al número de pagos, pero como en muchas ocasiones se asocia " $n$ " al número de años, el alumno se equivoca y pone el resultado expresado como unidades de tiempo. Por lo tanto, no se está dando un significado económico correcto al problema.

4.2 El resultado final no tiene mucho sentido aunque el procedimiento sea correcto.

En este caso se da por válido un resultado sin reflexionar sobre si este tiene o no sentido. Ejemplo:

Después de resolver el problema se establece que se obtiene una rentabilidad negativa del-7,3\% por adquirir y mantener una Letra del Tesoro durante un año (MF).

Esto no tiene sentido porque nadie adquiriría ningún activo financiero seguro si éste proporcionase una rentabilidad negativa.

Ejemplo:

En una cuenta de ahorro en la que se van realizando continuamente aportaciones y no se retira dinero, no es posible que el valor final acumulado sea inferior al valor inicial (GO). 
4.3 Proporcionan un resultado que no es el que se pide.

Ya sea porque no se sabe resolver lo que se pide, por falta de tiempo o porque no leen correctamente lo que están pidiendo en el enunciado.

Ejemplo:

Se pide que se resuelva el cuadro de amortización de un empréstito normal, y como resultado en vez de resolver el cuadro completo se presenta el valor de la anualidad del empréstito (MG)

Se puede decir que, en este apartado, aunque las soluciones sean correctas en sí mismas, no responden a la tarea de interpretación económica que requiere la misma, por lo que se podría considerar como soluciones no verificadas (Mosvshovitz-Hadar et al., 1987).

\section{Errores técnicos}

Incluimos aquí errores debidos al manejo incorrecto de símbolos algebraicos u originados en el cálculo de los resultados.

5.1 Error al despejar.

Ejemplo:

En mitad de un ejercicio se tiene la siguiente expresión $A=C-C \cdot d \cdot \frac{t}{360}$. Al intentar despejar $t$, se opera incorrectamente de la siguiente manera (GD): $t=\frac{A}{C-C \cdot d} \cdot 360$, mientras que lo correcto sería: $t=\frac{(C-A) \cdot 360}{C \cdot d}$.

5.2 Aplican el procedimiento correcto, pero no llegan a despejar la incógnita de la expresión resultante, o bien parten de una fórmula matemática complicada y no saben continuar.

En este caso los alumnos están resolviendo correctamente el problema pero en un momento determinado no saben cómo despejar algo de una fórmula y ponen "para finalizar se despeja de la ecuación la incógnita de la ecuación”. Esto ocurre, por ejemplo, cuando se les pide que calculen el momento de vencimiento del capital y no llegan a espejarlo.

Ejemplo:

Se pide en el ejercicio que se calcule el momento de vencimiento $t$, del capital $C$ sustituto de los capitales $\left(C_{1}\right.$ y $\left.C_{2}\right)$, de vencimientos respectivos $t_{1}$ y $t_{2}$, conociéndose para dicha operación la tasa de descuento simple $d(\mathrm{XR})$. En este caso se plantea correctamente la equivalencia de capitales $C_{1}\left(1-t_{1} \cdot d\right)+C_{1}\left(1-t_{1} \cdot d\right)=C(1-t \cdot d)$ pero luego no sabe despejar $t$ de dicha ecuación por lo que pone "de aqui se despeja ty obtendríamos el resultado del problema".

5.3 Problemas con el tratamiento de los paréntesis.

Estos errores corresponden a aquellos casos en los que el paréntesis presenta una dificultad especial. Ejemplo:

Se capitalizan los capitales $355310 €$ y 118888,06€ que se encuentran ambos en el momento inicial para conocer de cuánto dinero se dispondría dentro de dos años si se aplica una tasa de interés del 3,5\%. En el error (GD) se escribe $(1,035)^{2} \cdot 355310+118888,06$ 
Esa expresión es incorrecta porque de ese modo sólo se capitalizaría el primer capital y no ambos. Lo correcto sería:

$(1,035)^{2} \cdot(355310+118888,06)$, afectando así la capitalización a ambos capitales.

5.4 Fallo de cálculo en el resultado final.

El procedimiento es correcto pero se confunden en el cálculo final. Este problema está en muchas ocasiones muy relacionado con los dos anteriores.

Ejemplo:

En el error (VC) se escribe incorrectamente 7408,68€ cuando la respuesta correcta es: $17308,68 \cdot 0,945^{-14}=7408,68 €$.

Los errores situados en este apartado, como en el caso del apartado anterior, han sido también identificados en alumnos de secundaria como errores técnicos según Mosvshovitz-Hadar et al. (1987).

\section{b) Errores de origen e incidencia específicos}

Un resultado de este estudio de especial interés es la identificación de algunos errores que corresponden a determinados aspectos matemáticos y que afectan a contenidos económicos muy concretos.

En la tabla siguiente incluimos los diferentes apartados identificados en este segundo bloque $b$ ) y que representan el 27,33\% (82/300) de los errores, así como el número de errores correspondientes a cada uno de los subgrupos.

\begin{tabular}{|l|ll|r|}
\hline \multicolumn{3}{|c|}{ b) ERRORES DE ORIGEN E INCIDENCIAS ESPECÍfICOS } \\
\hline \multirow{2}{*}{$\begin{array}{l}\text { 1. Asociados a los Números Racio- } \\
\text { nales }\end{array}$} & 1.1 & Equivalencia de porcentajes y números decimales & 5 errores \\
\cline { 3 - 4 } & 1.2 & Despreciar decimales & 6 errores \\
\cline { 2 - 4 } & 1.3 & Significado de la fracción como operador & 57 errores \\
\hline 2. Asociados a la Magnitud Tiempo & 2.1 & $\begin{array}{l}\text { Relacionados con las equivalencias temporales y/o su expre- } \\
\text { sión simbólica posterior }\end{array}$ & \\
\hline
\end{tabular}

\section{Asociados a los Números Racionales.}

La mayoría de los errores situados en este apartado están vinculados de alguna manera a los números racionales.

1.1 Equivalencia de porcentajes y números decimales.

Este error afecta especialmente al tratamiento de los tantos por cien o por mil. Los errores aquí situados están asociados a las dificultades para ver que, por ejemplo, un $25 \%$ es lo mismo que $25 / 100$ ó 0,25 . De la misma manera, cuando el alumno tiene un tanto por mil expresado como $25 \%$, algunas veces asume erróneamente que es igual a 25/100 ó a 0,25.

Ejemplo:

Se establece en el ejercicio que existe una comisión del 0,3\% del nominal, siendo éste en este caso de $1500 €$. En la respuesta al ejercicio se pone $0,03 \cdot 1500=4,5 €$ de comisión (FS). 
El resultado es erróneo, ya que el 0,3\% es 0,003, y así en el ejercicio la comisión que se cobra es $4,5 €$ (esto es, $0,003 \cdot 1500=4,5 €$ ). En este sencillo ejercicio podemos observar cómo un problema en el cálculo de decimales lleva a establecer un pago de $45 €$ en vez de $4,5 €$, lo cual es una gran diferencia.

1.2 Despreciar decimales.

Este tipo de errores puede tener una indudable repercusión en el mundo financiero, ya que ignorar decimales, (por ejemplo, en el tipo de interés) puede afectar al resultado en grandes cantidades de dinero.

Ejemplo:

Se dice que por la imposición de un capital de $100.000 €$ durante 4 años una entidad financiera nos concede una tasa de interés anual compuesto de 4,75\%. En este caso se resuelve el ejercicio ignorando los decimales, considerando 0,04 en vez de 0,0475, quedando el resultado del ejercicio de la siguiente manera: $100000 \cdot(1,0475)^{4}=116985,85 €(\mathrm{GC})$.

Este resultado es erróneo, siendo el resultado correcto: $100000 \cdot(1,0475)^{4}=120397,13 €$. Aquí se aprecia que simplemente obviando esos decimales habríamos dejado de recibir en esta operación $3.411,28 €$ que es la diferencia entre 120397,13-116985,85=3.411,28€.

La principal causa de este error puede proceder de otras materias, ya que hasta que llegan a esta asignatura se les suele decir a los alumnos que consideren sólo dos decimales. Esto puede representar un obstáculo (Brousseau, 1983), especialmente en el caso de la asignatura matemáticas financieras donde suele tratarse con unidades monetarias. Un ejemplo claro de esta importancia es que no es lo mismo que nos concedan una hipoteca al 5\% de interés anual que al 5,58\% de interés anual.

1.3 Significado de la fracción como operador .

Estos errores surgen porque a menudo los alumnos no conocen o comprenden la labor del porcentaje como operador (algo que actúa sobre un número y lo modifica).

Ejemplo de los errores incluidos en este apartado:

Se pide en un problema que se calcule el precio de adquisición de una Letra del Tesoro que se adquiere a precio medio, siendo el precio medio de la letra un 93,5\% de su nominal que es $1000 €$, y no existe ningún tipo de comisión de adquisición. En este caso se da como solución errónea 0,935 (MF).

En este caso la solución correcta sería Precio Adquisición $=\frac{93,5}{100} \cdot 1000 \rightarrow 935 €$.

Los errores que hemos incluido en este apartado 1 pueden situarse en diferentes categorías de los autores mencionados anteriormente. Así, por ejemplo, todos ellos podrían ser asociados a la complejidad de los objetos matemáticos señalada por Socas (1997), o bien, siguiendo a MosvshovitzHadar et al. (1987) el último podría ser considerado dentro de los errores técnicos. Hemos querido presentarlos juntos porque nos parece importante resaltar como diferentes aspectos que tienen su origen en la comprensión de los números racionales pueden ser causa de errores relacionados con los porcentajes, muy utilizados en el mundo económico.

\section{Asociados a la Magnitud Tiempo.}

2.1 Relacionados con las equivalencias temporales y/o su expresión simbólica posterior.

Gran parte de los errores situados en este apartado se encuentran relacionados con el tiempo y las equivalencias temporales. 
- Equivalencias entre factores en un mismo producto u operación.

En este apartado situamos fundamentalmente problemas de equivalencias entre tasas de interés o descuento y la unidad de tiempo empleada en el ejercicio. Por ejemplo, en el enunciado de un ejercicio puede hacerse referencia a una tasa de interés trimestral compuesta para ser aplicada durante $\mathrm{n}$ años ( $\mathrm{i}_{4} ; \mathrm{n}$ años), o una tasa de descuento trimestral que será aplicada durante 5 años $\left(\mathrm{d}_{4} ; 5\right.$ años), o una tasa de interés cuatrimestral compuesta para ser aplicada durante 24 meses $\left(\mathrm{i}_{3}\right.$; 24 meses). En estos casos, para resolver el ejercicio el alumno ha de realizar las transformaciones necesarias para expresar todos los términos en la misma unidad temporal. Sin embargo, algunos fallan en este proceso.

Ejemplo:

Se dispone de una serie de capitales $\left(C_{1}, C_{2} y C_{3}\right)$ que vencen en los siguientes periodos de tiempo anuales respectivamente $\left(t_{1} ; t_{2} ; t_{3}\right)$ y se quieren descontar todos los capitales al momento inicial $\left(t_{0}\right)$ mediante una tasa de descuento simple trimestral $\left(d_{4}\right)$. Se pone incorrectamente en el error identificado como (RX):

$$
C=C_{1}\left(1-d_{4} \cdot 4 \frac{\left(t_{1}-t_{0}\right)}{360}\right)+C_{2}\left(1-d_{4} \cdot 4 \frac{\left(t_{2}-t_{0}\right)}{360}\right)+C_{3}\left(1-d_{4} \cdot 4 \frac{\left(t_{3}-t_{0}\right)}{360}\right)
$$

Lo correcto sería:

$$
C=C_{1}\left(1-d_{4}\left(t_{1}-t_{0}\right) 4\right)+C_{2}\left(1-d_{4}\left(t_{2}-t_{0}\right) 4\right)+C_{3}\left(1-d_{4}\left(t_{3}-t_{0}\right) 4\right)
$$

En este caso, el error puede ser debido a que el alumno no sabe cómo pasar de años a trimestres, y mezcla diferentes unidades temporales (trimestres y años). De manera general, el principal problema de este error se encuentra en que tienen dificultades para realizar equivalencias entre diferentes unidades temporales.

- Trabajar con valores que se encuentran en diferentes periodos temporales como si se encontrasen en un mismo periodo temporal.

Para poder comparar capitales financieros todos deben encontrarse en un mismo periodo temporal, es decir, no podemos comparar capitales situados en diferentes periodos salvo que realicemos su equivalencia en un determinado momento, ya que no es lo mismo pagar $5 €$ hoy que pagarlos dentro de un año. Todo debe compararse en un mismo momento temporal.

\section{Ejemplo:}

Se pide que se calcule el saldo o cantidad que queda disponible al final del centésimo mes, teniendo en cuenta que lo disponible será lo que quedaba pendiente en el mes cuadragésimo y en el sexagésimo. Para resolverlo se pone de manera errónea que el saldo disponible en dicho mes (saldo pendiente en ese momento) es igual al saldo del cuadragésimo mes más el saldo pendiente al final del sexagésimo mes. Esto es: $\mathrm{S}_{100}=\mathrm{S}_{60}+\mathrm{S}_{40}$

Esto no es correcto, ya que habría que trasladar el saldo del mes sexagésimo al mes centésimo (es decir, hacer su equivalencia empleando el tipo de interés vigente en el periodo de tiempo existente desde el mes 60 al 100) e igualmente para el caso del saldo del mes cuadragésimo (es decir, calcular su correspondiente valor equivalente en el mes centésimo en función de la tasa o tasas de interés aplicables desde el mes $40 \mathrm{al}$ mes 100). Una vez calculados sus valores equivalentes en ese momento es posible sumarlos y calcular el saldo pendiente en el centésimo mes. 
- Dificultades en expresar las diferencias temporales.

Es muy común en el mundo económico-empresarial tener que valorar pagos que suceden en determinados momentos temporales en otros diferentes. Estas diferencias temporales son muy importantes a la hora de resolver las situaciones planteadas y no considerarlas o considerarlas erróneamente conduce a equívocos como el que mostramos a continuación.

Ejemplo:

Se pretende sustituir dos capitales $\left(C_{1} y C_{2}\right)$ que serán pagados en los años $t_{1} y t_{2}$ por un único capital C cuyo pago se realizará en el año $t_{3}$ (siendo $t_{1}<t_{2}<t_{3}$ ), y se pide que se plantee la equivalencia financiera en ese último momento si el tipo de interés aplicado es del $6 \%$ anual. La respuesta errónea (identificada como BC) es: $C=C_{1}\left(1+0,06 \cdot t_{1}\right)+C_{2}\left(1+0,06 \cdot t_{2}\right)$

La correcta es: $C=C_{1}\left(1+0,06 \cdot\left(t_{3}-t_{1}\right)\right)+C_{2}\left(1+0,06 \cdot\left(t_{3}-t_{2}\right)\right)$

- Momento de valoración.

Causa también errores la dificultad que plantea el no conocer en qué momento se piden o valoran los capitales, como se ejemplifica a continuación.

Ejemplo:

Se pide que se calcule cuánto dinero tendremos ahorrado si realizamos 20 aportaciones anuales constantes de $100 €$ cada una de ellas, realizándose la primera al final del primer año, y el banco nos aplica una tasa de interés anual compuesta pospagable del 5\%. El error consiste en que se calcula cuánto dinero dispondremos hoy (valor actual) de la siguiente forma:

$$
100 \frac{1-(1+0,05)^{-20}}{0,05}=1.246,22 €
$$

Lo correcto es calcular el valor final, esto es, la cantidad acumulada que se calcula según la siguiente expresión:

$$
100 \frac{(1+0,05)^{20}-1}{0,05}=3.306,6 €
$$

Como ocurre en el apartado 1, los errores que hemos incluido en este apartado 2 pueden situarse en diferentes categorías de los autores anteriormente mencionados. Lo que nos parece importante destacar en este caso es cómo una magnitud como el tiempo (cuya génesis compleja ha sido ya indicada en trabajos como los de Piaget (1978) desarrollados en los primeros niveles infantiles) sigue presentando dificultades con posterioridad, afectando a contenidos económicos tan importantes como las equivalencias temporales entre tasas de interés y descuento, la valoración de capitales en diferentes periodos de tiempo, etc.

\section{ALGUNAS CONCLUSIONES E IMPLICACIONES}

Este estudio ha realizado una aproximación a los errores de los alumnos universitarios que cursan asignaturas relacionadas con las matemáticas financieras. En relación con los objetivos que nos habíamos planteado, podemos concluir que algunos de los errores matemáticos identificados están también presentes en los resultados de las investigaciones realizadas con alumnos de secundaria. Este estudio 
confirma la resistencia de estos errores y muestra como pueden afectar a muy diferentes aspectos matemático-financieros. Así, por ejemplo, los problemas que puede originar el lenguaje en matemáticas, tanto en relación con el vocabulario común como con las palabras específicamente matemáticas, han sido destacados por Socas (1987) e identificados en el trabajo de Movshovitz-Hadar et al. (1987). Su fuerte presencia en aspectos relacionados con las matemáticas financieras nos previene de su persistencia posterior.

Junto a ello, hemos identificado otros errores vinculados a algunos contenidos matemáticos que tienen incidencia en el desarrollo de ideas específicas de la materia. En particular, el estudio ha mostrado como los números racionales y la magnitud tiempo, los cuales juegan un papel muy importante en el desarrollo de ideas básicas en el campo de la matemática financiera, son causas frecuentes de errores en el alumnado.

Como profesores universitarios, el análisis realizado nos ha permitido ser conscientes de cómo estos errores pueden afectar el futuro trabajo profesional de nuestros alumnos, y su identificación desde nuestra práctica docente pensamos que puede ser objeto de un estudio posterior por los investigadores en el campo.

Queremos destacar que no pretendemos obtener más conclusiones con respecto a los errores identificados de las que se pueden extraer de un estudio interpretativo que no pretende en ningún caso generalizar. Asumimos que nuestros resultados están muy vinculados a un tipo concreto de problemas, enmarcados en un contexto universitario específico y con una muestra limitada. Sin embargo, pensamos que los resultados obtenidos pueden ayudar a los profesores universitarios a anticipar las dificultades que los mencionados errores pueden ocasionar y a prevenirlos de algún modo, así como a reducir las dificultades de comprensión en los contenidos de asignaturas de Economía y Organización de Empresas relacionadas con las matemáticas, señaladas por autores como Manchón et al. (2007). En cierta medida, este trabajo podría contribuir a ampliar la información proporcionada por los estudios anteriores, extendiéndola al campo de los estudios relacionados con las ciencias económicas y empresariales.

\section{REFERENCIAS BIBLIOGRÁFICAS}

Blanco, L. J., hidAlgo, M. J. y GARrote, M. (2004). Dificultades en el aprendizaje de las desigualdades e inecuaciones. Suma: Revista sobre Enseñanza y Aprendizaje de las Matemáticas, (46), pp. 37-44.

BROUSSEAU, G. (1983). Les obstacles épistémologiques et les problèmes en mathématiques. Recherches en Didactique des Mathématiques, 4 (2), 165-198.

FERNÁNDEZ, G.M. y ESCRIBANO, M.C. (2005). El primer eslabón de las matemáticas en las facultades de CC. Económicas y Empresariales: los análisis económicos. Revista Electrónica de Comunicaciones y Trabajos de ASEPUMA n ${ }^{\circ}$ Actas 13.

GONZÁLEZ-MARTÍN, A. y CAMACHO, M. (2005). Sobre la comprensión en estudiantes de matemáticas del concepto de integral impropia. Algunas dificultades, obstáculos y errores. Enseñanza de las Ciencias, 23 (1), pp. 81- 96.

GUILLEN, G. (2000). Sobre el aprendizaje de conceptos geométricos relativos a los sólidos. Ideas erróneas. Enseñanza de las Ciencias, 18(1), pp. 35-53.

MANCHÓN, C. y BLANCO, L. J. (2007). Economía y Matemáticas en el bachillerato: una visión desde el alumnado. Epsilon: Revista de la Sociedad Andaluza de Educación Matemática "Thales" (67), pp. $9-22$. 
MOVSHOVITZ-HADAR, N., ZASLAVSKY, O. y INBAR, S. (1987). An empirical classification model for errors in high school mathematics, Journal for Research in Mathematics Education, 18, 1, pp. 3-14. ORTEGA, J.F. y ORTEGA, J.A. (2001). Matemáticas ¿un problema del lenguaje?. Revista Electrónica de Comunicaciones y Trabajos de ASEPUMA no Actas 9.

PIAGET, J. (edición de 1978). El desarrollo de la noción de tiempo en el niño. Fondo de cultura económica: Argentina.

RICO, L. (1995). Errores en el aprendizaje de las Matemáticas. En J. Kilpatrick. P. Gómez y L. Rico (Edts.) Educación Matemática. GEI: Bogota.

SOCAS, M. (1997). Dificultades, obstáculos y errores en el aprendizaje de las matemáticas en la educación secundaria. En L. Rico (Coord.). La Educación Matemática en la Enseñanza Secundaria. ICE:Horsori. 


\title{
ERRORS IN LEARNING OF FINANCIAL MATHEMATICS
}

\author{
Ana F. Carazo, Raúl Brey \\ Departamento de Economía, Métodos Cuantitativos e Historia Económica. \\ Universidad Pablo de Olavide, Sevilla \\ afercar@upo.es•rbresan@upo.es
}

The incorporation of the Spanish universities into the European Space of Higher Education has given rise to a great change in university studies, which affects both the contents and the teaching methodology, explicitly highlighting the importance of the learning processes. Once the relevance of these processes in higher education has been assumed, it is necessary to investigate all the factors that can affect them. From this perspective, the students' errors could be regarded as «indicators» of what is happening in those processes.

This article focuses on the investigation of the mathematical errors made by university students who took a course on Financial Mathematics as part of their degree in Economics or Business Administration. In particular, our aims are:

- To identify common mathematical errors made by the students,

- To explore how these errors may affect concepts and procedures that are relevant in their professional field.

We want to point out that this study is not concerned in identifying students' lacks, but it looks for responsibilities in other educational levels. Instead, our purpose is to address the different errors that we observe in our university students from a double temporal perspective: past, looking for their causes in order to help students to deal with them, and future, identifying them as a possible source of problems in their later professional activity.

Within the different lines of research about identified errors, in this work we focus on the identification and classification of mathematical errors made by university students of Economics or Business Administration. Given that, in our case, we study what students do, we understand that the model proposed by Mosvshovitz-Hadar et al. (1987) provides an appropriate framework for a first approach.

The data sources were different exercises solved by the students in class in different academic years (2006/2007-2007/2008). We selected those exercises in which they made any type of error (one or more). These exercises were carefully reviewed, checking if the error was systematic (in the sense that a student made the same error several times in different exercises), and discarding those errors that could be considered as accidental. This way, we identified 300 errors that are the final data set used here.

We use an empirical-inductive approach for the analysis of the errors and their subsequent identification and classification, interpreting them later with respect to the knowledge of financial mathematics that is intended to develop. Our approach can be decomposed into a three-step process: analysis of the identified systematic errors in a qualitative way, grouping of these errors based on their similarity following the model provided by Mosvshovitz-Hadar et al (1987), and review of each of the obtained groups, according to how they might affect the future professional activity of the students.

The results have shown that some of the identified errors are also found in studies carried out with secondary students, confirming their persistence and revealing their effects on different aspects of the subject of Financial Mathematics. In addition, we have identified other errors linked to contents such as the rational numbers and the temporal dimension, which may affect the understanding of basic ideas in the considered field of study.

As university teachers, these results have allowed us to be aware of how these errors can affect the future professional activity of our students. We think that their identification from the teaching practice may be a subject of a deeper future study by researchers in this field.

We would like to emphasize that we do not intend to obtain conclusions with respect to the identified errors beyond those that can be reasonably drawn from an interpretative study. We know that our results are contingent on the particular type of problem being addressed, the framing of the study, and the reduced sample size. Therefore, these results cannot be generalised. However, we think that our results may help academics to know in advance and prevent the difficulties that the identified errors may cause. 
